\title{
Blinking model and synchronization in small-world networks with a time-varying coupling
}

\author{
Igor V. Belykh $^{\mathrm{a}, *}$, Vladimir N. Belykh ${ }^{\mathrm{b}}$, Martin Hasler ${ }^{\mathrm{a}}$

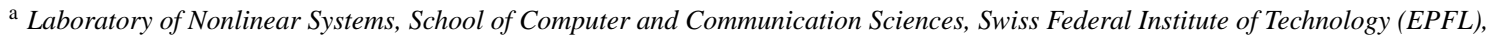 \\ CH-1015 Lausanne, Switzerland \\ b Mathematics Department, Volga State Academy, 5, Nesterov st., Nizhny Novgorod 603 600, Russia
}

Received 25 April 2003; received in revised form 24 February 2004; accepted 17 March 2004

\begin{abstract}
The paper proposes a new type of small-world networks of cells with chaotic behavior. This network consists of a regular lattice of cells with constant $2 K$-nearest neighbor couplings and time-dependent on-off couplings between any other pair of cells. In each time interval of duration $\tau$ such a coupling is switched on with probability $p$ and the corresponding switching random variables are independent for different links and for different times. At each moment, the coupling structure corresponds to a small-world graph, but the shortcuts change from time interval to time interval, which is a good model for many real-world dynamical networks. It is to be distinguished from networks that have randomly chosen shortcuts, fixed in time. Here, we apply the Connection Graph Stability method, developed in the companion paper ("Connection graph stability method for synchronized coupled chaotic systems", see this issue), to the study of global synchronization in this network with the time-varying coupling structure, in the case where the on-off switching is fast with respect to the characteristic synchronization time of the network. The synchronization thresholds are explicitly linked with the average path length of the coupling graph and with the probability $p$ of shortcut switchings in this blinking model. We prove that for the blinking model, a few random shortcut additions significantly lower the synchronization threshold together with the effective characteristic path length. Short interactions between cells, as in the blinking model, are important in practice. To cite prominent examples, computers networked over the Internet interact by sending packets of information, and neurons in our brain interact by sending short pulses, called spikes. The rare interaction between arbitrary nodes in the network greatly facilitates synchronization without loading the network much. In this respect, we believe that it is more efficient than a structure of fixed random connections.
\end{abstract}

(C) 2004 Elsevier B.V. All rights reserved.

PACS: 05.45.-a; 05.45.Xt

Keywords: Small-world networks; Blinking model; Synchronization; Stability; Averaging

\footnotetext{
* Corresponding author. Tel.: +41-21-693-27-34; fax: +41-21-693-67-00.

E-mail address: igor.belykh@epfl.ch (I.V. Belykh).
} 


\section{Introduction and motivation for the model}

In 1967, the social psychologist Milgram discovered the small-world phenomenon (popularly known as six degrees of separation) [1]. He concluded that six was the average number of acquaintances separating any two people in the world. In 1998, Watts and Strogatz proposed a semi-random network, called a small-world [2]. This network is a regular coupled lattice (the "pristine world") having a few additional randomly arising long-range shortcuts. They conjectured that dynamical systems coupled in this way would display enhanced propagation speed, synchronizability and computation power, as compared with regular lattices of the same size [3]. This model has received a great deal of attention in the physical and mathematical literature [3-25]. The small-world effect has been shown to be widespread in many human generated networks as well as biological networks, including the WWW connections [9,10], social and scientific networks [3,11-13], epidemiological models [14-16], electrical power grids [2,17], electronic circuits [18] and neural and biochemical networks [19,20].

Most theoretical studies were concerned with statistical and combinatorical properties of small-world networks where the cells do not have individual temporal dynamics [3-8]. Dynamical processes on small-world networks were studied relatively little and mainly by means of computer simulation. In particular, it was shown numerically that small-world connections may drastically improve synchronization properties of networks of limit-cycle and chaotic oscillators [17,20-24]. In turn, synchronization in networks of periodic and chaotic oscillators with different regular and random coupling configurations has been intensively studied. ${ }^{1}$

More recently, significant progress in the study of the relation between the addition of random shortcuts and the synchronization properties of networks was made by Barahona and Pecora [25]. They applied the Master Stability function approach [27] to the study of local synchronization in small-world networks and showed, through numerics and analysis, how the addition of random shortcuts improves network synchronizability. The connection matrix $G$ was initially chosen at random, and then fixed forever. This is the usual approach to defining the small-world networks. Within this approach, the statistics of the connection matrices $G$ were translated into the statistics of the synchronization thresholds.

In this paper, we study small-world networks where the shortcuts change as a function of time. Instead of randomly choosing the shortcuts and leaving them fixed, we randomly choose the shortcuts, leave them fixed for an interval of time $\tau$, and then randomly choose another set of shortcuts, leave them again for a lapse of time $\tau$ fixed, etc. More precisely, our probabilistic model is the following. During each time interval of length $\tau$, every possible shortcut is turned on with probability $p$, independently of the switching on and off of the other shortcuts, and independently of whether or not it has been turned on during the previous time interval. Furthermore, we assume that the switching time $\tau$ is small with respect to the characteristic synchronization/desynchronization time of the coupled system.

This way of transforming a network with fixed couplings, the "pristine world", into a time-varying small-world network can always be applied. We call it the "blinking model". In this paper, we shall concentrate on the important example where the pristine world is a ring of $2 K$-nearest neighbor coupled chaotic oscillators. The methods developed here, however, are more generally applicable. In this paper, we also introduce and study a purely random "blinking" network, where the fixed connections of the pristine world are absent and the short on-off interactions arise between any pair of cells.

The blinking model is indeed of practical importance. In practice, often collections of subsystems that are organized into a network actually interact only sporadically. This is true in biology as well as in technology. Neurons in the brain send out electrical signals in the form of spikes and most of the interaction with the other neurons takes place during the arrival of the spikes at the connection points, the synapses. Since the spike duration

\footnotetext{
${ }^{1}$ For the detailed description of the concept of chaotic synchronization and literature on synchronization in regular and random networks, the reader is addressed to the companion paper [26].
} 
is usually small with respect to the interspike intervals, this is an important example of "blinking" interaction. In fact, to fit the blinking model with the pristine world where the coupling is always on, the neural network must be organized (and this is often true in reality) by both electrical and chemical synapses. In this case, the electrical couplings (gap junctions) provide the constant local couplings between the neurons within the pristine world, and the chemical ones (pulse couplings) ensure the global pulsatile connections such that they are responsible for the blinking "random" interactions. At the same time, the purely random blinking model relates to networks of neurons having only chemical synapses. Of course, the model is rather abstract, and the occurrence of spikes of different neurons and at different times are not just independent random variables, and the spike durations are actually caused by the dynamics of the individual neurons. Nevertheless, the distant node interaction is of intermittent nature.

In technology, practical systems exist that can be modeled rather precisely by the blinking model. Packet switched networks such as the Internet are an important example. Dynamical processes in the computers that are networked through Internet interact by sending messages that are subdivided into packets and sent over the network. Both in the network links and the computers themselves, they have to share the available communication time slots with many other packets belonging to communications between different computers and/or different processes. The occurrence of the other packets can be considered as independent, and the timeslots available for the communication between specific processes can also often be considered independent, due to the congestion of the links by the other packets. Thus, the blinking model may be appropriate in many different situations.

To refer to synchronization more specifically, let us remark the following. Processes may interact in order to achieve many different goals, but in order to be able to interact in a controlled way, they need to refer to a common time reference. In other words, the clocks that generate the local time for the computer need to be synchronized throughout the network. This is done by sending information about each other's time as packets through the network. In the Internet system, this is done by means of the Network Time Protocol (NTP) used by time servers and their clients to synchronize clocks, as well as automatically organize and maintain the time synchronization subnet itself [28]. In NTP, one or more primary servers synchronize directly to external reference sources (national standards) by wire, radio or calibrated atomic clocks. In practice, each NTP server synchronizes with several other servers in order to mitigate outages and to increase precision. The limiting factor in the time accuracy of the local computer is the stability of the local clock, which is usually implemented by an uncompensated quartz oscillator (a good temperature-compensated oscillator is a relatively expensive component not likely to be found in cost-competitive computers). The synchronizing signals (packets), that aim at reducing the timing errors, should be sufficiently frequent in order to guarantee a sufficient precision of synchronization between the clocks, but, on the other hand, the network should not be overloaded by these "administrative" messages. This is a trade-off between the precision of synchronization and the traffic load on the network. To some extent, this frequent "blinking" network administration is a way to provide reliable and precise functioning of the network composing of nonprecise elements. The packets arriving at a node are used to control the local clock by means of Phase-Lock loops. The details of this control can be found in [28,29].

Another example of switched, i.e. "blinking", networks of practical importance are pulse-width modulated power converters [30].

The main objective of this paper is to study the synchronization properties of the small-world blinking model of coupled periodic or chaotic oscillators. Applying the Connection Graph Stability method, developed in the companion paper [26], we obtain a rigorous bound for the coupling constant required to achieve global synchronization and link it with probability $p$ of shortcut switchings. This result shows that only a small probability $p$ for shortcuts is necessary to decrease the threshold coupling strength for complete synchronization in the blinking model.

The layout of this paper is as follows. First, in Section 2, we describe the blinking model and derive the averaged model. Then, in Section 3, we study synchronization in this averaged model which is obtained by adding to the pristine world (the ring of $2 K$-nearest neighbor coupled oscillators) an additional global coupling. We show that this global coupling addition significantly improves the synchronization properties of the network and give a 
rigorous bound for the coupling strength necessary for synchronization of all the oscillators. In Section 4, we show that the study of the blinking model dynamics can be relieved by averaging such that the threshold for complete synchronization in the blinking model is the same as the threshold in the averaged model for almost all (all, except for a set of probability zero) instances of the fast shortcut switching random process. It is proven for the blinking model that a few random shortcut additions significantly lower the synchronization threshold together with the effective characteristic path length. The synchronization properties of the blinking small-world model are compared with small-world networks with randomly chosen but fixed shortcuts, and it is shown that the blinking interaction provides more reliable synchronization. Finally, a brief discussion of the obtained results is given.

\section{Small-world networks with a time-varying coupling and the blinking model}

The model of small-world networks that we propose consists of the pristine world (the regular locally coupled lattice of oscillators) and a time-dependent on-off coupling between any other pair of cells.

Here we shall use the same notations for the networks of coupled oscillators as we have used in the companion paper [26]. Hence we consider the network

$$
\dot{x}_{i}=F\left(x_{i}\right)+\sum_{j=1}^{n} \varepsilon_{i j}(t) P_{\mathrm{c}} x_{j}, \quad i=1, \ldots, n,
$$

where $x_{i}=\left(x_{i}^{1}, \ldots, x_{i}^{d}\right)$ is the $d$-vector of the coordinates of the $i$ th oscillator. The $n \times n$ connection matrix $G(t)=$ $\left(\varepsilon_{i j}(t)\right)$ is symmetric and has vanishing row-sums and nonnegative off-diagonal elements such that $\varepsilon_{i j}=\varepsilon_{j i}, \varepsilon_{i j} \geq 0$ for $i \neq j$, and $\varepsilon_{i i}=-\sum_{j=1 ; j \neq i}^{n} \varepsilon_{i j}, i=1, \ldots, n$.

The matrix $P_{\mathrm{c}}$ determines by which variables the oscillators are coupled. For simplicity, we assume that the individual systems are only connected through the first state variable $x^{1}$, i.e. the matrix

$$
P_{\mathrm{c}}=\left(\begin{array}{ll}
1 & 0 \\
0 & 0
\end{array}\right),
$$

where the first line and column correspond to the first state variable and the remaining lines and columns to the state variables $x^{2}$ to $x^{d}$. The generalization of all the results of the paper to connections between more than one, or even all state variables is straightforward.

As the pristine world, we take a conventional network, a ring of $2 K$-nearest neighbor coupled oscillators. In this case, the connection matrix $G$, corresponding to the blinking model, has the $2 K$ adjacent diagonals with the coupling constants $\varepsilon$ and on-off time-dependent small-world connections parameters $\varepsilon_{i_{r} j_{r}}(t)$ standing in all remaining places of the matrix $G$, where $r=1,2, \ldots, n(n-2 K-1) / 2$.

To define the functions $\varepsilon_{i_{r} j_{r}}(t)$ we divide the time axis into intervals of length $\tau$. We assume $\varepsilon_{i_{r} j_{r}}(t)$ to be binary signals that take the constant value $\varepsilon$ with probability $p$ and the value 0 with probability $1-p$ in each time interval. Thus, the blinking model is a dynamical system combined with a probability space $(\Omega, \Sigma, P)$ that is defined as follows.

An elementary event $\omega \in \Omega$ is a semi-infinite sequence of binary vectors of dimension $n(n-2 K-1) / 2$, i.e.

$$
\omega=(\mathbf{s}(1), \mathbf{s}(2), \ldots)=\left(\begin{array}{ccc}
s_{1, K+2}(1) & s_{1, K+2}(2) & \ldots \\
s_{1, K+3}(1) & s_{1, K+3}(2) & \ldots \\
\vdots & \vdots & \\
s_{n-K-1, n}(1) & s_{n-K-1, n}(2) & \ldots
\end{array}\right),
$$



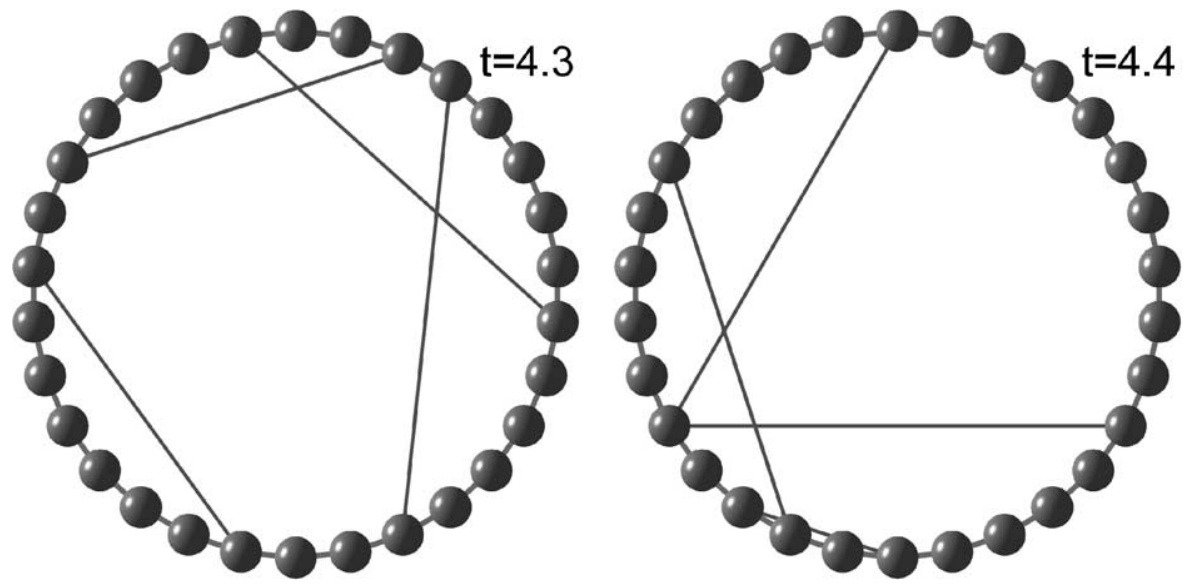

Fig. 1. The blinking model of shortcuts connections. Probability of switchings $p=0.01$, the switching time step $\tau=0.1$.

where $s_{i j}(q) \in\{0,1\}$. The $\sigma$-algebra $\Sigma$ of events is generated by the sets

$$
\Omega_{i j}^{1}(q)=\left\{\omega \mid s_{i j}(q)=1\right\}, \quad \Omega_{i j}^{0}(q)=\left\{\omega \mid s_{i j}(q)=0\right\}
$$

and the probability $P$ is defined by

$$
P\left(\Omega_{i j}^{1}(q)\right)=p, \quad P\left(\Omega_{i j}^{0}(q)\right)=1-p
$$

and the requirement that the events $\Omega_{i j}^{\sigma}(q)$ are independent for different $i, j$ and/or $q$. As usual, to the quantities $s_{i j}(q)$ the random variables $S_{i j}(q)$ are associated, defined by

$$
\left[S_{i j}(q)\right](\omega)=s_{i j}(q) .
$$

By hypothesis, all these random variables are independent and identically distributed.

Fig. 1 shows the time-varying structure of shortcut connections in the blinking model of 30 coupled systems.

The meaning of $\omega$ is a switching sequence, i.e. $\omega$ indicates which of the remaining (nonpristine) links that are turned at a given time interval. Thus, the time-dependent coupling constants are random variables defined by

$$
\left[\varepsilon_{i j}(t)\right](\omega)= \begin{cases}\varepsilon & \text { if nodes } i \text { and } j \text { are connected in the pristine world, } \\ \varepsilon s_{i j}(q) & \text { if not, and if }(q-1) \tau<t<q \tau,\end{cases}
$$

where $q$ numbers the time intervals.

The mean values of $\varepsilon_{i j}$ for the remaining (shortcut) links are

$$
\bar{\varepsilon}_{i j}=\lim _{t \rightarrow \infty} \frac{1}{t} \int_{0}^{t} \varepsilon_{i j}(u) \mathrm{d} u=\varepsilon \lim _{q \rightarrow \infty} \frac{1}{Q} \sum_{q=1}^{Q} S_{i j}(q) .
$$

By the strong law of large numbers, for almost all switching sequences $\omega$,

$$
\bar{\varepsilon}_{i j}=\varepsilon p \text {. }
$$

We will show in Section 4 that, for almost all switching sequences, the threshold for complete synchronization in the blinking model is the same as the threshold in the averaged model, where the remaining links are constant, with value $\varepsilon p$. This property holds under the condition that the switching is fast enough compared with the transient time of synchronization/desynchronization phenomenon. An explicit expression for this condition will be given. The proof only concerns bounds on the synchronization thresholds, not the thresholds themselves, even though the authors believe that the correspondence between the blinking model and the averaged model is much more general. 
Before starting the rigorous analysis of the blinking model, we study the synchronization properties of the averaged model.

\section{Auxiliary regular coupling scheme: the averaged model}

We consider the regular coupled network by adding to the pristine world (the ring of $2 K$-nearest neighbor coupled oscillators) an additional global coupling such that the coupling coefficient $p \varepsilon$ is added to all free places of the matrix $G, 0 \leq p \leq 1$. In this case, the network acquires an all-to-all coupling configuration with two different coupling strengths $\varepsilon$ and $p \varepsilon$.

Note that in this auxiliary model, contrary to the blinking model, the parameter $p$ is not a probability.

Fig. 2 illustrates this coupling scheme (for $K=1$ ) which for an arbitrary $K$ gives the averaged system matrix $G_{\text {mean }}$

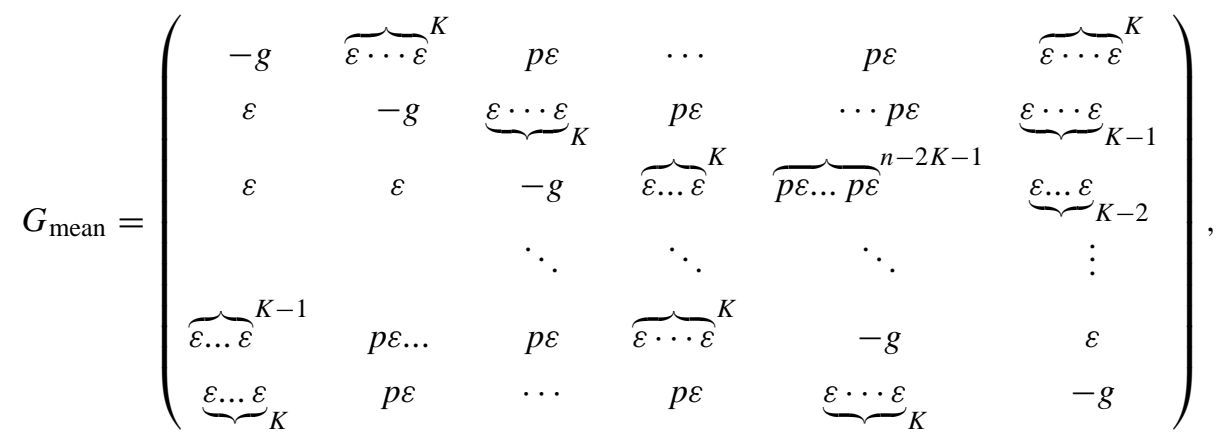

where $g=\varepsilon[2 K+p(n-2 K-1)]$ such that it preserves vanishing row-sums.

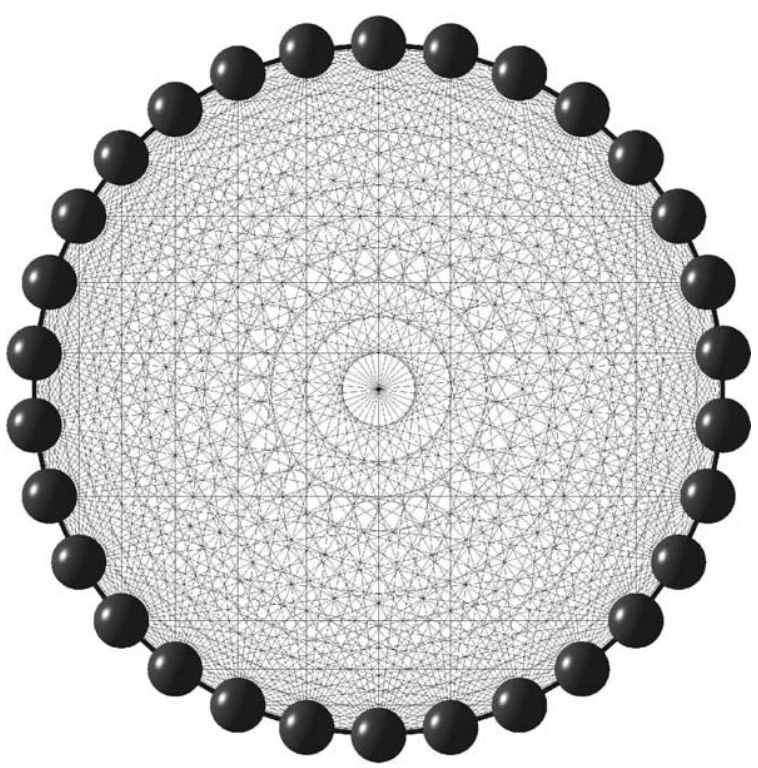

Fig. 2. All-to-all coupled network: the pristine world (here, $K=1$ ) with the local coupling strength $\varepsilon$ and the additional global coupling $p \varepsilon$. Here, $p$ is small, such that the width of the links may be thought of as the coupling strength (a strong coupling within the pristine world and a weak coupling for the remaining all-to-all links). 
In the matrix $G_{\text {mean }}$, the $2 K$ adjacent diagonals represent the pristine world (a ring of $2 K$-nearest neighbor coupled oscillators) with the coupling $\varepsilon$. The remaining $n-2 K-1$ adjacent diagonals define the additional global coupling with the coupling $p \varepsilon$.

The use of this auxiliary coupling scheme is two-fold. First, the study of global synchronization in such a network is interesting in itself, and allows us to show once more how effective the Connection Graph Stability method is. Second, it represents the averaged model for the blinking model such that its synchronization properties are directly connected with the stability of the synchronous state in the blinking model.

The main objective of this section is to determine the threshold value for the coupling strength required for synchronization in this all-to-all globally coupled network with the coupling matrix $G_{\text {mean }}$, and to reveal its dependence on the number of oscillators and the structure of the network. In particular, the main question under study is how the threshold coupling depends on the parameter $p$.

We start with the main equality of the Connection Graph Stability method [26] which provides the following criterion for complete synchronization in this auxiliary network with matrices $G_{\text {mean }}$ and $P_{\mathrm{c}}$

$$
\sum_{l=1}^{K} \sum_{i=1}^{n} \varepsilon\left(X_{i, i+l \bmod n}^{1}\right)^{2}+\sum_{l=K+1}^{[n / 2]} \sum_{i=1}^{n} p \varepsilon\left(X_{i, i+l \bmod n}^{1}\right)^{2}>\frac{a}{n} \sum_{i=1}^{n-1} \sum_{j>i}^{n}\left(X_{i j}^{1}\right)^{2} .
$$

Here, as in the companion paper [26], $X_{i j}^{1}=x_{j}^{1}-x_{i}^{1}, i, j=1, \ldots, n$ are the difference variables, and the stability parameter $a$ is defined by the individual oscillator dynamics and the matrix $P_{\mathrm{c}}$. As before, we consider only networks that admit global synchronization and for which the parameter $a$ can be derived. Once again, we have supposed that the coupling between the oscillators is only through the first variable $x^{1}$ but a generalization to more than one is easy.

This inequality is similar to that of the main example (the ring of $2 K$-nearest neighbor coupled oscillators) of the companion paper except that the additional sum (the second left-hand side term) is present. This term provides the contribution of the additional all-to-all coupling links.

To get rid of the presence of the dynamical difference variables in inequality (10) and hence to obtain the synchronization threshold $\varepsilon^{*}$, we should bound the sum $\sum_{i=1}^{n-1} \sum_{j>i}^{n}\left(X_{i j}^{1}\right)^{2}$ by the sum

$$
\left[\sum_{l=1}^{K} \sum_{i=1}^{n}\left(X_{i, i+l \bmod n}^{1}\right)^{2}+p \sum_{l=K+1}^{[n / 2]} \sum_{i=1}^{n}\left(X_{i, i+l \bmod n}^{1}\right)^{2}\right]
$$

That is, we have to determine $b(n, K, p)$ such that

$$
\sum_{i=1}^{n-1} \sum_{j>i}^{n}\left(X_{i j}^{1}\right)^{2} \leq b(n, K, p)\left[\sum_{l=1}^{K} \sum_{i=1}^{n}\left(X_{i, i+l \bmod n}^{1}\right)^{2}+p \sum_{l=K+1}^{[n / 2]} \sum_{i=1}^{n}\left(X_{i, i+l \bmod n}^{1}\right)^{2}\right] .
$$

From this, it will follow that the synchronization condition becomes

$$
\varepsilon>\varepsilon^{*}=\frac{a}{n} b(n, K, p) .
$$

Now, we have to calculate $b(n, K, p)$.

Adding and subtracting the additional $\operatorname{sum} b(n, K, p) \cdot p \sum_{l=1}^{K} \sum_{i=1}^{n}\left(X_{i, i+l \bmod n}^{1}\right)^{2}$ from the right part of inequality (11), and taking into account the trivial equality 


$$
p \sum_{l=1}^{K} \sum_{i=1}^{n}\left(X_{i, i+l \bmod n}^{1}\right)^{2}+p \sum_{l=K+1}^{[n / 2]} \sum_{i=1}^{n}\left(X_{i, i+l \bmod n}^{1}\right)^{2}=p \sum_{i=1}^{n-1} \sum_{j>i}^{n}\left(X_{i j}^{1}\right)^{2},
$$

we obtain

$$
\sum_{i=1}^{n-1} \sum_{j>i}^{n}\left(X_{i j}^{1}\right)^{2} \leq b(n, K, p)\left[(1-p) \sum_{l=1}^{K} \sum_{i=1}^{n}\left(X_{i, i+l \bmod n}^{1}\right)^{2}+p \sum_{i=1}^{n-1} \sum_{j>i}^{n}\left(X_{i j}^{1}\right)^{2}\right] .
$$

Consequently, we have

$$
\sum_{i=1}^{n-1} \sum_{j>i}^{n}\left(X_{i j}^{1}\right)^{2} \leq \frac{b(n, K, p)(1-p)}{1-p b(n, K, p)} \sum_{l=1}^{K} \sum_{i=1}^{n}\left(X_{i, i+l \bmod n}^{1}\right)^{2} .
$$

But we have proved in the companion paper [26] (cf. Example: ring of $2 K$-nearest neighbor coupled oscillators) that

$$
\sum_{i=1}^{n-1} \sum_{j>i}^{n}\left(X_{i j}^{1}\right)^{2} \leq b(n, K) \sum_{l=1}^{K} \sum_{i=1}^{n}\left(X_{i, i+l \bmod n}^{1}\right)^{2},
$$

where $b(n, K)=(n / 2 K)^{3}(1+(65 / 4)(K / n))$. Therefore, it is sufficient to choose $b(n, K, p)$ such that

$$
\frac{b(n, K, p)(1-p)}{1-p b(n, K, p)}=b(n, K)
$$

which is equivalent to

$$
b(n, K, p)=\frac{b(n, K)}{1+p(b(n, K)-1)} .
$$

Hence, we finally come to the following assertion.

Theorem 1. The upper bound for global synchronization in the averaged network with the connection matrix $G_{\text {mean }}$ is

$$
\varepsilon>\varepsilon^{*}=\frac{a}{n} \frac{b(n, K)}{1+p(b(n, K)-1)} .
$$

Since, for the pristine world, the depth of nearest-neighbor interaction $K$ is usually much smaller than the number of nodes $n(K \ll n)$, we can write $b(n, K) \cong(n / 2 K)^{3}=L^{3}(0)$, where $L(0)=n /(2 K)$ is the average path length of the coupling graph corresponding to the pristine world.

Therefore, we can rewrite the criterion (13) as

$$
\varepsilon>\varepsilon^{*} \cong \frac{a}{n} \frac{L(0)}{1+p(L(0)-1)} .
$$

One can see from Eq. (14) that the threshold $\varepsilon^{*}$ decreases rapidly as a function of the parameter $p$.

As before, we conjecture that for networks for which the global stability parameter $a$ can be derived, the real conditions for total synchronization obey the same laws of dependence on the number of oscillators $n$, the range of the pristine world $K$, and the parameter $p$, but with a lower constant than $a$.

We checked numerically the dependence (14) on $p$ for the network (9) of $30 x$-coupled Lorenz systems. Fig. 3 (top) shows the synchronization thresholds $\varepsilon^{*}$ calculated numerically for different $p$ (depicted by small circles). 

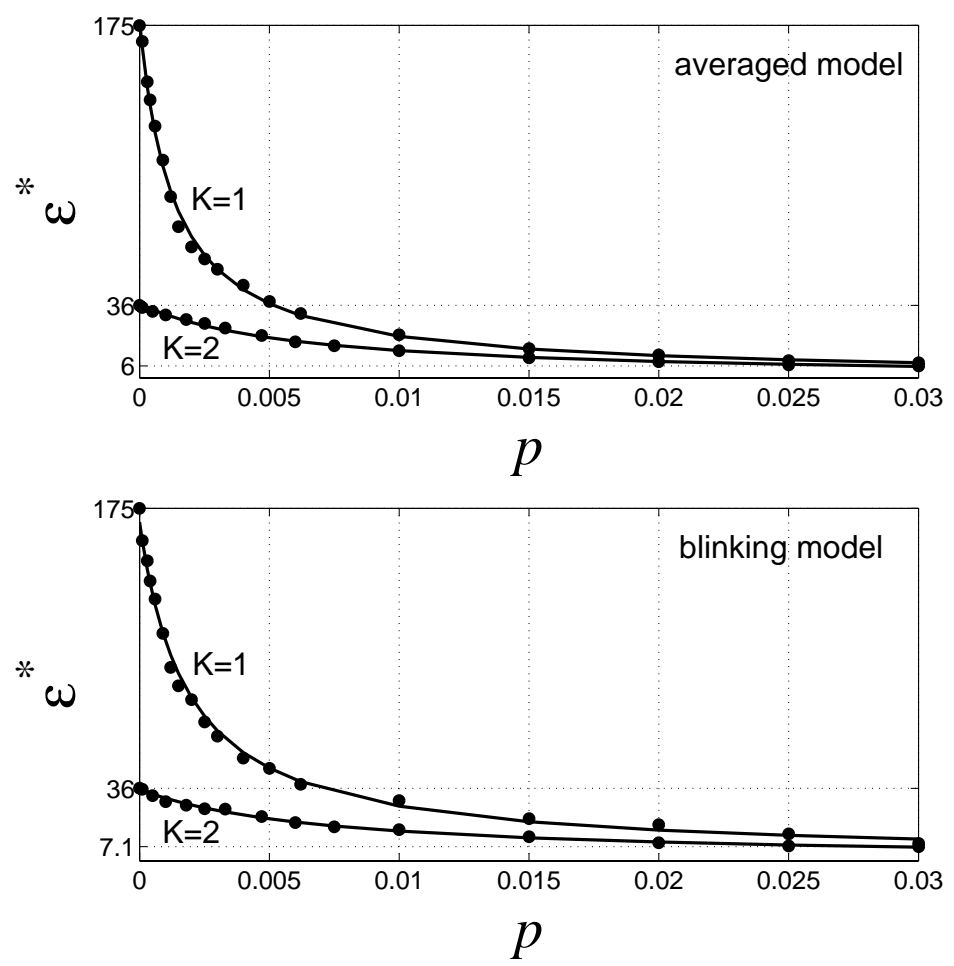

Fig. 3. Dependence of the synchronization thresholds on the parameter $p$ in the averaged network with the coupling matrix $G_{\text {mean }}$ (top) and on the probability $p$ of the shortcut appearance in the blinking model (bottom). The pristine world is a ring of $302 K$-nearest neighbor coupled Lorenz systems. The switching time in the blinking model $\tau=0.1$. The analytical curve $\varepsilon^{*}=(a / n) L(0) /[1+p(L(0)-1)]$ (solid line) fits the numerical data (small circles) remarkably well.

The analytical estimate (14) is fitted to the numerical data by adapting the constant $a$. The resulting curve fits the data remarkably well. This numerical example confirms also that only a very weak additional all-to-all coupling is required to significantly reduce the synchronization threshold and therefore to improve the synchronization properties of the network.

After having studied synchronization in the averaged network, we can tackle the problem of synchronization in the blinking model.

\section{The blinking model: averaging and synchronization}

In order to analyze the synchronization behavior of the blinking model, we use the old technique of averaging that was developed for oscillating dynamical systems [31]. As in the companion paper [26], we consider the Lyapunov function

$$
V=\frac{1}{4} \sum_{i, j=1}^{n} X_{i j}^{\mathrm{T}} \cdot H \cdot X_{i j},
$$

where $H$ is a suitable positive definite matrix (cf. Hypothesis 2). Due to our assumption that the individual oscillators are coupled through the first state variable, and more precisely, through the difference variable $X_{i j}^{1}=x_{j}^{1}-x_{i}^{1}, i, j=$ 
$1, \ldots, n$, and the matrices $H$ and $A$ (cf. [26]) have the form

$$
H=\left(\begin{array}{cc}
h & 0 \\
0 & \tilde{H}
\end{array}\right), \quad A=\left(\begin{array}{cc}
a & 0 \\
0 & 0
\end{array}\right),
$$

where once again the first line and column correspond to the first state variable and the remaining lines and columns to the state variables $x^{2}$ to $x^{d}$, and $h$ and $d$ are suitable positive constants. Since $H$ is positive definite, its smallest eigenvalue $h_{\min }$ is also positive.

In order to prove that $V(t)$ converges to zero, and therefore to prove the stability of synchronization in the blinking model, we need a few hypotheses. Considering the individual system dynamics, we make the same basic hypotheses (Hypotheses 1 and 2) as in [26].

Hypothesis 1. The individual systems are eventually dissipative, which implies that there is a compact invariant set $B$ such that, after some finite time, every solution is in $B$.

Due to the nature of the coupling, the solutions of the coupled system also reach the compact invariant set $\tilde{B}=B \times B \times \cdots \times B$ in finite time. On this set, all state variables and derivatives of state variables are bounded. In particular, we deduce from the state equations for the difference variables

$$
\dot{X}_{i j}=\left[\int_{0}^{1} D F\left(\beta x_{j}+(1-\beta) x_{i}\right) \mathrm{d} \beta\right] X_{i j}+\sum_{k=1}^{n}\left\{\varepsilon_{j k} P_{\mathrm{c}} X_{j k}-\varepsilon_{i k} P_{\mathrm{c}} X_{i k}\right\}
$$

that

$$
\begin{aligned}
|\dot{V}| & \leq \frac{1}{2}\left|\sum_{i, j=1}^{n} X_{i j}^{\mathrm{T}} H\left[\int_{0}^{1} D F\left(\beta x_{j}+(1-\beta) x_{i}\right) \mathrm{d} \beta\right] X_{i j}\right|+\frac{1}{2}\left|\sum_{i, j, k=1}^{n} X_{i j}^{1} h\left\{\varepsilon_{j k} X_{j k}^{1}-\varepsilon_{i k} X_{i k}^{1}\right\}\right| \\
& \leq \frac{C}{2} \sum_{i, j=1}^{n}\left\|X_{i j}\right\|^{2}+\frac{\varepsilon}{2} \sum_{i, j, k=1}^{n}\left|X_{i j}^{1}\right| h\left\{\left|X_{j k}^{1}\right|+\left|X_{i k}^{1}\right|\right\},
\end{aligned}
$$

where $C=\|H\| \max _{x \in B}\|D F(x)\|$. Using the Cauchy-Schwarz inequality, we obtain

$$
\begin{aligned}
|\dot{V}| & \leq \frac{C}{2 h_{\min }} \sum_{i, j=1}^{n} X_{i j}^{\mathrm{T}} H X_{i j}+\frac{\varepsilon}{2} \sqrt{n \sum_{i, j=1}^{n} h\left(X_{i j}^{1}\right)^{2}} \sqrt{n \sum_{j, k=1}^{n} h\left(X_{j k}^{1}\right)^{2}}+\frac{\varepsilon}{2} \sqrt{n \sum_{i, j=1}^{n} h\left(X_{i j}^{1}\right)^{2}} \sqrt{n \sum_{i, k=1}^{n} h\left(X_{i k}^{1}\right)^{2}} \\
& \leq \frac{1}{T_{\text {syn }}} V(t),
\end{aligned}
$$

where

$$
T_{\text {syn }}=\left(\frac{C}{2 h_{\min }}+\frac{n \varepsilon}{2}\right)^{-1}=\left(\frac{\|H\| \max _{x \in B}\|D F(x)\|}{2 h_{\min }}+\frac{n \varepsilon}{2}\right)^{-1}
$$

can be interpreted as the characteristic synchronization/desynchronization time constant of the coupled system.

Given the positivity of $V(t)$, this implies for $t_{2}>t_{1}$

$$
V\left(t_{1}\right) \mathrm{e}^{-\left(t_{2}-t_{1}\right) / T_{\text {syn }}} \leq V\left(t_{2}\right) \leq V\left(t_{1}\right) \mathrm{e}^{\left(t_{2}-t_{1}\right) / T_{\text {syn }}} .
$$

Hypothesis 2. On the set $B$, the positive matrix $H$ defines a quadratic Lyapunov function $W=(1 / 2) X^{\mathrm{T}} H X$ for the difference variables of the individual system dynamics corrected by the term $-A X$. The derivative of this Lyapunov function along any pair of solutions $x(t), y(t)$ of the individual systems satisfies, 


$$
\dot{W}=X^{\mathrm{T}}\left[H\left(\int_{0}^{1} D F(\beta y+(1-\beta) x) \mathrm{d} \beta-A\right)\right] X \leq \frac{1}{T_{\text {stab }}} X^{\mathrm{T}} H X
$$

for some constant $T_{\text {stab }}$, where $X(t)=y(t)-x(t)$.

In [26], only $\dot{W} \leq 0$ was required, which is slightly weaker than condition (22). The constant $T_{\text {stab }}$ can be interpreted as the characteristic time for the solutions of the stabilized individual system to converge to each other. Note that it depends on the choice of $A$.

As shown in [26], the time derivative of the Lyapunov function $V$ has the form

$$
\dot{V}=\frac{1}{4} \frac{\mathrm{d}}{\mathrm{d} t}\left(\sum_{i, j=1}^{n} X_{i j}^{\mathrm{T}} H X_{i j}\right)+\frac{1}{2}\left(\sum_{i, j=1}^{n} X_{i j}^{\mathrm{T}} A X_{i j}\right)-\frac{1}{2} \sum_{i, j, k=1}^{n}\left\{\varepsilon_{j k} X_{j i}^{\mathrm{T}} H P_{\mathrm{c}} X_{j k}+\varepsilon_{i k} X_{i k}^{\mathrm{T}} H P_{\mathrm{c}} X_{i j}\right\}
$$

and, combined with condition (22),

$$
\dot{V} \leq-\frac{2}{T_{\text {stab }}} V(t)+\sum_{i, j=1 ; j>i}^{n}\left[a-n \varepsilon_{i j}(t)\right] h\left(X_{i j}^{1}\right)^{2}
$$

Using inequality (11) obtained for the averaged system (1)-(9) and taking into account that $\varepsilon_{i j}(t)=\varepsilon$ for the pristine world links, we obtain

$$
\begin{aligned}
\dot{V} \leq & -\frac{2}{T_{\text {stab }}} V(t)+a b(n, K, p)\left[\sum_{l=1}^{K} \sum_{i=1}^{n} h\left(X_{i, i+l \bmod n}^{1}\right)^{2}+p \sum_{l=K+1}^{[n / 2]} \sum_{i=1}^{n}\left(X_{i, i+l \bmod n}^{1}\right)^{2}\right] \\
& -n \varepsilon \sum_{l=1}^{K} \sum_{i=1}^{n} h\left(X_{i, i+l \bmod n}^{1}\right)^{2}-n \sum_{l=K+1}^{[n / 2]} \sum_{i=1}^{n} \varepsilon_{i, i+l \bmod n}(t) h\left(X_{i, i+l \bmod n}^{1}\right)^{2} .
\end{aligned}
$$

We now hypothesize that the averaged system synchronizes.

Hypothesis 3. The coupling between the oscillators is made sufficiently strong such that the condition for the bound on the synchronization threshold (13) in the averaged system holds, i.e.

$$
\varepsilon>\frac{a}{n} \frac{b(n, K)}{1+p(b(n, K)-1)}=\frac{a}{n} b(n, K, p) .
$$

Under this hypothesis, we can write

$$
\dot{V} \leq-\frac{2}{T_{\text {stab }}} V(t)-n \sum_{l=K+1}^{[n / 2]} \sum_{i=1}^{n}\left(\varepsilon_{i, i+l \bmod n}(t)-p \frac{a}{n} b(n, K, p)\right) h\left(X_{i, i+l \bmod n}^{1}\right)^{2} .
$$

While in previous applications of the Connection Graph Stability method, we required the expression in parenthesis be positive for any time $t$, Hypothesis 3 only requires a positivity in the mean.

We consider now inequality (27) in integral form, over some time interval $[t, t+\Delta t]$, where $t$ is large enough that the solution has reached the compact set $\tilde{B}$, and $\Delta t$ is to be specified later, 


$$
\begin{aligned}
V(t+\Delta t)-V(t) \leq & -\frac{2}{T_{\text {stab }}} \int_{t}^{t+\Delta t} V(u) \mathrm{d} u \\
& -n \sum_{l=K+1}^{[n / 2]} \sum_{i=1}^{n} \int_{t}^{t+\Delta t}\left[\varepsilon_{i, i+l \bmod n}(u)-p \frac{a}{n} b(n, K, p)\right] h\left(X_{i, i+l \bmod n}^{1}(u)\right)^{2} \mathrm{~d} u \\
= & -\frac{2}{T_{\text {stab }}} \int_{t}^{t+\Delta t} V(u) \mathrm{d} u \\
& -n \sum_{l=K+1}^{[n / 2]} \sum_{i=1}^{n} \int_{t}^{t+\Delta t}\left[\varepsilon_{i, i+l \bmod n}(u)-p \frac{a}{n} b(n, K, p)\right] \times h\left(X_{i, i+l \bmod n}^{1}(t)\right)^{2} \mathrm{~d} u \\
& -n \sum_{l=K+1}^{[n / 2]} \sum_{i=1}^{n} \int_{t}^{t+\Delta t}\left[\varepsilon_{i, i+l \bmod n}(u)-p \frac{a}{n} b(n, K, p)\right] \\
& \times h\left\{\left(X_{i, i+l \bmod n}^{1}(u)\right)^{2}-\left(X_{i, i+l \bmod n}^{1}(t)\right)^{2}\right\} \mathrm{d} u \\
\leq & -\frac{2}{T_{\text {stab }}} \int_{t}^{t+\Delta t} V(u) \mathrm{d} u \\
& -n \sum_{l=K+1}^{[n / 2]} \sum_{i=1}^{n} h\left(X_{i, i+l \bmod n}^{1}(t)\right)^{2} \int_{t}^{t+\Delta t}\left[\varepsilon_{i, i+l \bmod n}(u)-p \frac{a}{n} b(n, K, p)\right] \mathrm{d} u \\
& +n \varepsilon \sum_{l=K+1}^{[n / 2]} \sum_{i=1}^{n} \int_{t}^{t+\Delta t} h\left|\left(X_{i, i+l \bmod n}^{1}(u)\right)^{2}-\left(X_{i, i+l \bmod n}^{1}(t)\right)^{2}\right| \mathrm{d} u .
\end{aligned}
$$

Now,

$$
\frac{1}{2} \frac{\mathrm{d}}{\mathrm{d} t} h\left(X_{i j}^{1}\right)^{2}=h X_{i j}^{1}\left[\int_{0}^{1} D F\left(\beta x_{j}+(1-\beta) x_{i}\right) \mathrm{d} \beta\right] X_{i j}+h X_{i j}^{1} \sum_{k=1}^{n}\left\{\varepsilon_{j k} P_{\mathrm{c}} X_{j k}^{1}-\varepsilon_{i k} P_{\mathrm{c}} X_{i k}^{1}\right\}
$$

and thus

$$
\frac{1}{2}\left|\frac{\mathrm{d}}{\mathrm{d} t} h\left(X_{i j}^{1}(s)\right)^{2}\right| \leq C\left\|X_{i j}\right\|^{2}+\varepsilon \sum_{k=1}^{n} h\left|X_{i j}^{1}\right|\left\{\left|X_{j k}^{1}\right|+\left|X_{i k}^{1}\right|\right\},
$$

where again $C=\left\|H \mid \max _{x \in B}\right\| D F(x) \|$. Therefore, using the same derivation as for inequality (19), we get

$$
\begin{aligned}
& \sum_{l=K+1}^{[n / 2]} \sum_{i=1}^{n} h\left|\left(X_{i, i+l \bmod n}^{1}(u)\right)^{2}-\left(X_{i, i+l \bmod n}^{1}(t)\right)^{2}\right| \\
& \quad \leq \frac{1}{2} \sum_{i, j=1}^{n} h\left|\left(X_{i j}^{1}(u)\right)^{2}-\left(X_{i j}^{1}(t)\right)^{2}\right| \leq \frac{1}{2} \int_{t}^{u} h \sum_{i, j=1}^{n}\left|\frac{\mathrm{d}}{\mathrm{d} t} h\left(X_{i j}^{1}(s)\right)^{2}\right| d s \\
& \quad \leq \int_{t}^{u}\left(C \sum_{i, j=1}^{n}\left|\left(X_{i j}(s)\right)\right|^{2}+\varepsilon \sum_{i, j, k=1}^{n} h\left|X_{i j}^{1}(s)\right|\left\{\left|X_{j k}^{1}(s)\right|+\left|X_{i k}^{1}(s)\right|\right\}\right) \mathrm{d} s \leq-\frac{1}{T_{\mathrm{syn}}} \int_{t}^{u} V(s) \mathrm{d} s .
\end{aligned}
$$

Inequality (31) leads to the following bound on the last term in inequality (28) 


$$
\begin{aligned}
& \left|n \varepsilon \sum_{l=K+1}^{[n / 2]} \sum_{i=1}^{n} \int_{t}^{t+\Delta t} h\right|\left(X_{i, i+l \bmod n}^{1}(u)\right)^{2}-\left(X_{i, i+l \bmod n}^{1}(t)\right)^{2}|\mathrm{~d} u| \\
& \quad \leq n \varepsilon\left(\int_{t}^{t+\Delta t}\left(\int_{t}^{u} \frac{V(s)}{T_{\mathrm{syn}}} \mathrm{d} s\right) \mathrm{d} u\right) \leq n \varepsilon \frac{\Delta t}{T_{\mathrm{syn}}} \int_{t}^{t+\Delta t} V(u) \mathrm{d} u .
\end{aligned}
$$

The second term in inequality (28) is expanded as follows

$$
\begin{aligned}
& -n \sum_{l=K+1}^{[n / 2]} \sum_{i=1}^{n} \int_{t}^{t+\Delta t}\left[\varepsilon_{i, i+l \bmod n}(u)-p \frac{a}{n} b(n, K, p)\right] h\left(X_{i, i+l \bmod n}^{1}(t)\right)^{2} \mathrm{~d} u \\
& =-n \sum_{l=K+1}^{[n / 2]} \sum_{i=1}^{n} \int_{t}^{t+\Delta t}\left[\varepsilon_{i, i+l \bmod n}(u)-p \varepsilon\right] h\left(X_{i, i+l \bmod n}^{1}(t)\right)^{2} \mathrm{~d} u-n p V_{1}(t)\left[\varepsilon-\frac{a}{n} b(n, K, p)\right] \Delta t,
\end{aligned}
$$

where

$$
V_{1}(t)=\sum_{l=K+1}^{[n / 2]} \sum_{i=1}^{n} h\left(X_{i, i+l \bmod n}^{1}(t)\right)^{2} \leq V(t) .
$$

Suppose now that $t=Q_{1} \tau, \Delta t=Q \tau$. Using the switching random variables, we obtain

$$
\int_{t}^{t+\Delta t}\left(\varepsilon_{i, i+l \bmod n}(u)-p \varepsilon\right) \mathrm{d} u=\tau \varepsilon \sum_{q=Q_{1}+1}^{Q_{1}+Q}\left(S_{i, i+l \bmod n}(q)-p\right)=\frac{\Delta t \varepsilon}{Q} \sum_{q=Q_{1}+1}^{Q_{1}+Q}\left(S_{i, i+l \bmod n}(q)-p\right) .
$$

As long as $(1 / Q) \sum_{q=Q_{1}+1}^{Q_{1}+Q}\left(S_{i, i+l \bmod n}(q)-p\right)$ is positive, its contribution to the derivative of the Lyapunov function $V$ is negative. If its value is slightly negative, we will still be able to prove that the Lyapunov function decreases overall during the time interval $\Delta t$, even though it may at some instants temporarily increase. If, however, its value is too close to its minimum $-\gamma, V$ may increase during the time interval $\Delta t$. We now bound the probability of the set of switching sequences where this last case takes place by a Chernoff bound $[32,33]$

$$
P\left(\frac{1}{Q} \sum_{q=Q_{1}+1}^{Q_{1}+Q}\left(S_{i, i+l \bmod n}(q)-p\right) \leq-\gamma p\right) \leq \mathrm{e}^{-Q p \gamma^{2} / 2}=\mathrm{e}^{-\left(p \gamma^{2} / 2\right) \Delta t / \tau},
$$

where $\gamma$ is any constant between 0 and 1, to be chosen later. Consequently, if we define the following set of switching sequences

$$
\Omega\left(Q_{1}, Q, \gamma, i, l\right)=\left\{\omega \mid \frac{1}{Q} \sum_{q=Q_{1}+1}^{Q_{1}+Q}\left(S_{i, i+l \bmod n}(q)-p\right) \leq-\gamma p\right\}
$$

for $1 \leq i \leq n, K+1 \leq l \leq[n / 2]$, then

$$
P\left(\Omega\left(Q_{1}, Q, \gamma, i, l\right)\right) \leq \mathrm{e}^{-\left(p \gamma^{2} / 2\right) \Delta t / \tau} .
$$

If we define by

$$
\Omega\left(Q_{1}, Q, \gamma\right)=\bigcup_{l=K+1}^{[n / 2]} \bigcup_{i=1}^{n} \Omega\left(Q_{1}, Q, \gamma, i, l\right)
$$


the set of all switching sequences whose average value for the samples between $Q_{1}+1$ to $Q_{1}+Q$ is lower than $(1-\gamma) p$ for at least one of its components, we obtain

$$
P\left(\Omega\left(Q_{1}, Q, \gamma\right)\right) \leq \frac{n^{2}}{2} \mathrm{e}^{-\left(p \gamma^{2} / 2\right) \Delta t / \tau}
$$

and for any switching sequence $\omega \notin \Omega\left(Q_{1}, Q, \gamma\right)$ we get

$$
\left|\sum_{l=K+1}^{[n / 2]} \sum_{i=1}^{n} \int_{t}^{t+\Delta t}\left[\varepsilon_{i, i+l \bmod n}(u)-p \varepsilon\right] h\left(X_{i, i+l \bmod n}^{1}(t)\right)^{2} \mathrm{~d} u\right| \leq V_{1}(t) \Delta t \varepsilon \gamma p .
$$

Combining inequality (28) with inequalities (32), (33) and (41), we obtain, under the conditions:

(1) $t \geq t_{0}$, where $t_{0}$ is the time at which the solution reaches the compact set $\tilde{B}$ and subsequently remains in it;

(2) $\Delta t=\tau Q$;

(3) $\omega \notin \Omega\left(Q_{1}, Q, \gamma\right)$;

the following estimate on the decrease of the Lyapunov function $V$ during the time interval $\Delta t$

$$
\begin{aligned}
V(t+\Delta t)-V(t)= & -\frac{2}{T_{\text {stab }}} \int_{t}^{t+\Delta t} V(u) \mathrm{d} u+n \varepsilon \frac{\Delta t}{T_{\text {syn }}} \int_{t}^{t+\Delta t} V(u) \mathrm{d} u+n V_{1}(t) \Delta t \varepsilon \gamma p \\
& -n p V_{1}(t)\left(\varepsilon-\frac{a}{n} b(n, K, p)\right) \Delta t .
\end{aligned}
$$

Now we can make suitable choices of the free constants $\Delta t$ and $\gamma$. We can set these constants as follows:

$$
\Delta t=\frac{T_{\text {syn }}}{n \varepsilon T_{\text {stab }}}, \quad \gamma=\frac{p}{\varepsilon}\left(\varepsilon-\frac{a}{n} b(n, K, p)\right) .
$$

With this choice of $\Delta t$ and $\gamma$, we get

$$
V(t+\Delta t)-V(t) \leq-\frac{1}{T_{\text {stab }}} \int_{t}^{t+\Delta t} V(u) \mathrm{d} u<0 .
$$

Using the lower bound of inequality (21) in the integral, we get

$$
V(t+\Delta t) \leq V(t)-\frac{V(t)}{T_{\text {stab }}} \int_{t}^{t+\Delta t} e^{-(u-t) / T_{\text {syn }}} \mathrm{d} u=V(t)\left(1-\frac{T_{\text {syn }}}{T_{\text {stab }}}\left(1-\mathrm{e}^{-1 / n \varepsilon T_{\text {stab }}}\right)\right) .
$$

Setting

$$
\alpha_{\text {contr }}=-\ln \left(1-\frac{T_{\text {syn }}}{T_{\text {stab }}}\left(1-\mathrm{e}^{-1 / n \varepsilon T_{\text {stab }}}\right)\right), \quad \alpha_{\text {exp }}=\frac{\Delta t}{T_{\text {syn }}}=\frac{1}{n \varepsilon T_{\text {stab }}},
$$

we conclude from inequalities (45) and (21) that

$$
V\left(Q_{1} \tau+Q \tau\right) \leq \begin{cases}V\left(Q_{1} \tau\right) \mathrm{e}^{\alpha_{\exp }} & \text { if } \omega \in \Omega\left(Q_{1}, Q, \gamma\right), \\ V\left(Q_{1} \tau\right) \mathrm{e}^{-\alpha_{\text {contr }}} & \text { if } \omega \notin \Omega\left(Q_{1}, Q, \gamma\right) .\end{cases}
$$

This is true for any $Q_{1} \geq Q_{0}=t_{0} / \tau$, where $t_{0}$ is the time when the solution reaches the compact invariant set $\tilde{B}$. The number $Q$, on the other hand, is fixed by

$$
Q=\frac{\Delta t}{\tau}=\frac{T_{\text {syn }}}{\tau} \frac{1}{n \varepsilon T_{\text {stab }}} .
$$


Now, we define the new random variables $\tilde{S}_{m}, m=0,1,2, \ldots$ by

$$
\tilde{S}_{m}(\omega)= \begin{cases}\alpha_{\exp } & \text { if } \omega \in \Omega\left(Q_{0}+m Q, Q, \gamma\right), \\ -\alpha_{\text {contr }} & \text { if } \omega \notin \Omega\left(Q_{0}+m Q, Q, \gamma\right) .\end{cases}
$$

Since they are based on different switching times for different $m$, they are independent. They are equally distributed, with common mean value $\tilde{E}$. Furthermore,

$$
V\left(Q_{0} \tau+M \tau\right) \leq V\left(Q_{0} \tau\right) \mathrm{e}^{M\left((1 / M) \sum_{m=0}^{M-1} \tilde{S}_{m}\right)} .
$$

By the strong law of large numbers, for almost all switching sequences,

$$
\frac{1}{M} \sum_{m=0}^{M-1} \tilde{S}_{m} \rightarrow \tilde{E} \quad \text { for } M \rightarrow \infty
$$

This implies that for $M \rightarrow \infty$

$$
V\left(Q_{0} \tau+M \tau\right) \rightarrow 0 \quad \text { if } \tilde{E}<0
$$

for almost all switching sequences. Thanks to condition (21) this finally implies for almost all switching sequences that for $t \rightarrow \infty$

$$
V(t) \rightarrow 0 \quad \text { if } \tilde{E}<0 .
$$

Now we have to express the condition $\tilde{E}<0$. Using condition (40), we obtain

$$
\begin{aligned}
\tilde{E} & =\alpha_{\exp } P\left(\Omega\left(Q_{0}, Q, \gamma\right)\right)-\alpha_{\text {contr }}\left(1-P\left(\Omega\left(Q_{0}, Q, \gamma\right)\right)\right. \\
& =-\alpha_{\text {contr }}+\left(\alpha_{\text {exp }}-\alpha_{\text {contr }}\right) P\left(\Omega\left(Q_{0}, Q, \gamma\right)\right) \leq-\alpha_{\text {contr }}+\left(\alpha_{\text {exp }}-\alpha_{\text {contr }}\right) \frac{n^{2}}{2} \mathrm{e}^{-\left(p \gamma^{2} / 2\right) \Delta t / \tau} \\
& =-\alpha_{\text {contr }}+\left(\alpha_{\text {exp }}-\alpha_{\text {contr }}\right) \frac{n^{2}}{2} \mathrm{e}^{-\left(p \gamma^{2} / 2\right)\left(T_{\text {syn }} / \tau\right)\left(1 / n \varepsilon T_{\text {stab }}\right)}
\end{aligned}
$$

and the condition becomes

$$
\left(\frac{\alpha_{\text {exp }}}{\alpha_{\text {contr }}}-1\right) \frac{n^{2}}{2} \mathrm{e}^{-\left(p \gamma^{2} / 2\right)\left(T_{\text {syn }} / \tau\right)\left(1 / n \varepsilon T_{\text {stab }}\right)}<1 .
$$

Collecting all hypotheses and all inequalities, we obtain the main theorem for the synchronization bounds in the blinking model (1) and their connection to the bounds in the averaged system (1)-(9).

Theorem 2. Consider the blinking model described by

$$
\dot{x}_{i}=F\left(x_{i}\right)+\sum_{j=1}^{n} \varepsilon_{i j}(t)\left(x_{j}^{1}-x_{i}^{1}\right), \quad i=1, \ldots, n,
$$

where $x_{i}=\left(x_{i}^{1}, \ldots, x_{i}^{d}\right), F: R^{n} \rightarrow R^{n}$ is a $C^{1}$-function, $\varepsilon_{i j}(t)=\varepsilon_{j i}(t)=\varepsilon>0$ for $|j-i| \bmod n \leq K$ and all $t$, and $\varepsilon_{i j}(t)=\varepsilon_{j i}(t)=\varepsilon S_{i j}(q)$ for $(q-1) \tau \leq t<q \tau$ for all remaining pairs $(i, j)$. Here, $S_{i j}(q)$ is a random variable that takes the value 1 with probability $p$ and 0 with probability $1-p$.

The random variables $S_{i j}(q)$ are assumed to be all independent. To each realization $\omega$ of this stochastic process $S(1), S(2), \ldots$, where $S(q)=\left\{S_{i j}(q), i=1, \ldots n, j=i+l \bmod n, l=K+1, \ldots,[n / 2]\right\}$, i.e. to each switching sequence $\omega$, there corresponds a time-varying system described by Eq. (56). 
Suppose in addition that the network satisfies Hypotheses 1-3.

Then for a sufficiently small switching period $\tau$, and for almost all switching sequences, the system synchronizes completely for any choice of initial conditions.

More precisely, synchronization for almost all switching sequences takes place if the switching period $\tau$ is such that the inequality

$$
\left(\frac{\alpha_{\text {exp }}}{\alpha_{\text {contr }}}-1\right) \frac{n^{2}}{2} \mathrm{e}^{-\left(p \gamma^{2} / 2\right)\left(T_{\text {syn }} / \tau\right)\left(1 / n \varepsilon T_{\text {stab }}\right)}<1
$$

is satisfied, where $\alpha_{\mathrm{contr}}$ and $\alpha_{\exp }$ are given by Eq. (46), $\gamma$ by Eq. (43), $T_{\mathrm{syn}}$ by Eq. (20) and $T_{\mathrm{stab}}$ in Hypothesis 2.

Remark 1. The switching period appears only in the denominator of the exponent in condition (57). Therefore, the left-hand side of Eq. (46) decreases rapidly when $\tau$ decreases and the condition can always be satisfied for small enough $\tau$.

Remark 2. What matters is that $\tau$ is small enough compared to $T_{\text {sync }}$, i.e. that the switching is rapid with respect to the synchronization/desynchronization time.

Remark 3. The parameter $a$ is somewhat artificial since it is not directly given by the problem, but it is needed in the proof. If we choose $a$ close to the limit such that Hypothesis 2 is satisfied, $T_{\text {stab }}$ will be large, and switching will have to be rapid in order to guarantee synchronization. The same is true, if we choose $\varepsilon$ just slightly above $(a / n) b(n, K, p)$ which causes $\gamma$ to be small. In fact, for a sufficiently large fixed $\varepsilon$, there is a certain margin for $a$, and an optimal value could be sought in view of guaranteeing synchronization for switching as slow as possible. We have not performed this optimization.

To conclude, Theorem 2 proves that for almost all (all, except for a set of probability zero) instances of the fast switching random process, when the switching time $\tau$ is rapid with respect to the synchronization time $T_{\text {sync }}$ of the network, the threshold for complete synchronization in the blinking model is the same as the threshold in the averaged model with the matrix $G_{\text {mean }}$, where the remaining links are constant, with value $p$. Therefore, for sufficiently small $\tau / T_{\text {syn }}$ (cf. condition (57)) and

$$
\varepsilon>\varepsilon^{*}=\frac{a}{n} \frac{L^{3}(0)}{1+p\left(L^{3}(0)-1\right)}
$$

for almost all switching sequences, complete synchronization of all the oscillators in the blinking model is globally stable. Here, the average path length in the pristine world $(L(0))$ is $L(0)=n / 2 K$.

For $p=0$, the estimate (58) becomes the synchronization threshold for the pristine world, and for $p=1$, it gives the synchronization threshold for all-to-all coupling. For $0<p<1$, the dependence (58) of the synchronization threshold on $p$ reveals the reduction of the synchronization threshold such that the addition of a few small-world connections (small $p$ ) significantly improves the synchronization properties of the network (see Fig. 3 (bottom)).

In Fig. 3 (bottom), we see the synchronization thresholds $\varepsilon^{*}$ calculated numerically for the blinking network of $30 x$-coupled chaotic Lorenz systems, depicted by small circles. The curve $\varepsilon^{*}=(a / n) L(0) /[1+p(L(0)-1)]$ (solid line) is fitted to the numerical data, by letting $a$ vary. It can be seen that the deviation of the data from the fitted curve is very small. Thus, the blinking interactions arising even with a very small probability significantly lower the synchronization threshold. For example, for $p=0.01$ and $K=1$ (cf. Fig. 1) the threshold is reduced from 175 to about 29. 
Let us now introduce the effective path length for our blinking model and its dependence on the probability $p$. Recall that for $p=0$, the threshold (58) becomes the synchronization threshold for the ring of $2 K$-nearest neighbor coupled oscillators [26]

$$
\varepsilon>\varepsilon^{*}=\frac{a}{n} L^{3}(0) .
$$

Rewriting the dependence (58) in the form similar to Eq. (59), we introduce the effective path length of our model of dynamical small worlds as follows:

$$
L^{3}(p)=\frac{L^{3}(0)}{1+p\left(L^{3}(0)-1\right)} .
$$

Therefore, the normalized effective path length has the following dependence on the probability $p$

$$
\frac{L(p)}{L(0)}=\frac{1}{\left(1+\left(n^{3} / 8 k^{3}-1\right) p\right)^{1 / 3}} .
$$

This formula clearly manifests the significant decrease of the characteristic path length under a small increase of $p$ from 0 in the blinking model.

Remark 4. It is interesting to compare the small-world blinking model with the purely random blinking network, where the fixed interactions of the pristine world are absent. If we introduce the effective path length for the purely random blinking network, similarly to Eq. (60), we obtain $L(p)=1 / p$. Thus, in this case, again the effective path length decreases with $p$ (for $p=0$, this network is not connected and the effective average path is infinite).

However, the synchronization properties of the purely random blinking network are very poor. Indeed, the synchronization threshold for such a network is

$$
\varepsilon^{*}=\frac{a}{p n} .
$$

For small $p$, the network can synchronize only under a very strong coupling because only very few oscillators are connected at any given time. Moreover, the time needed for synchronization is large.

This is in contrast to the small-world blinking model where all oscillators will synchronize under a moderate coupling as long as condition (58) is satisfied, even if $p$ is very small. In fact, the pristine world connections guarantee a connected graph of all nodes at all times.

Let us now discuss the synchronization properties of the blinking small-world model with small-world networks with randomly chosen but fixed shortcuts that were studied numerically in [17,20-25]. Once again, the parameter $p$ in the blinking model represents the average fraction of time any shortcut connection is turned on. It also represents the average fraction of shortcuts that are turned on at any given time.

In previous papers on synchronization in small-world networks, a fraction of shortcuts is initially chosen at random and the shortcuts remain fixed for the rest of the time. In such an approach the synchronization threshold is the mean value of the thresholds for all possible shortcut combinations. However, these thresholds strongly depend on the particular choice of the shortcuts. It was stated in $[21,23,24]$ that a sufficient amount of randomly chosen shortcuts will cause total synchronization. In other words, there exists a critical value of the probability $p$ for which the small-world network, obtained by adding any given shortcuts, will synchronize completely. This statement is not always correct. It was shown in [25] that the addition of small-world links fixed in time does not necessarily guarantee synchronizability. In fact, the addition of links filling out an entire row in the coupling matrix $G$ produces a tremendous increase of connectivity and a sharp reduction of the synchronization threshold. At the same time, the 
addition of coupling coefficients, located in the matrix $G$ as a dense small "spot" and forming an all-to-all coupling within a small subgroup, does not substantially reduce the synchronization threshold. The latter case is not very likely to happen when there are many cells but it has nonetheless a nonzero probability for a finite number of cells.

On the contrary, when the critical probability $p$ is reached in the blinking model, then almost surely the system will synchronize, even for a finite number of cells. In other words, the set of on-off shortcut switching sequences that fail to force total synchronization has probability zero. For this property to be true, necessarily the switching time $\tau$ must be much smaller than $T_{\text {syn }}$, the characteristic synchronization time of the network. This allows the use of averaging. In this context, for many technical applications, and, probably, for the coordinating brain functions, the blinking effect of the shortcut appearance provides more reliable synchronization and global coordinating properties than the networks with the small-world but fixed coupling structure.

\section{Conclusions}

A new type of dynamical small-world networks of chaotic cells has been proposed. For the first time for such networks with a time-varying coupling configuration, mathematically rigorous bounds on the strength of the coupling between the cells have been established that are sufficient to achieve complete synchronization independently of the initial conditions.

Calculated by means of the Connection Graph Stability method, the synchronization thresholds have been explicitly linked with the average path length of the coupling graph and with the probability $p$. It shows the effectiveness of our general synchronization approach, and once again clearly indicates the connection between graph theory and network dynamics which makes intuitive sense. It is proven for the blinking model that a few random shortcut additions significantly lower the synchronization threshold together with the effective characteristic path length.

We have shown that interactions between arbitrary nodes in a network that are only sporadic, and of a short duration, are very efficient for achieving synchronization. In fact, the blinking interaction provides more reliable synchronization than the networks with the small-world fixed coupling structure. The authors believe that this will be used in various applications. Actually, interactions that are turned on and off is the mode of operation of packet-switched networks. Biological systems also use this method of communication/interaction. Indeed, neurons interact through electrical pulses, called spikes.

\section{Acknowledgements}

IB and MH acknowledge the financial support of the Swiss National Science Foundation through Grant N 2100-065268. We are grateful to Christopher Jones, who made an excellent choice of peer reviewers whose criticism and suggestions helped to improve the quality of both the companion papers. We are also thankful to Oscar De Feo and Christopher Cianci for valuable suggestions. The research was supported in part by INTAS (Grant No. 01-2061) and RFFI (Grant No. 02-01-00968).

\section{References}

[1] S. Milgram, Psychol. Today 2 (1961) 60.

[2] D.J. Watts, S.H. Strogatz, Nature 393 (1998) 440.

[3] S.H. Strogatz, Nature 410 (2001) 268.

[4] M. Barthélémy, L.A.N. Amaral, Phys. Rev. Lett. 82 (1999) 3180.

[5] R. Monasson, Eur. Phys. J. B 12 (1999) 555. 
[6] M.E.J. Newman, J. Stat. Phys. 101 (2000) 819.

[7] M.E.J. Newman, C. Moore, D.J. Watts, Phys. Rev. Lett. 84 (2000) 3201.

[8] M.E.J. Newman, S.H. Strogatz, D.J. Watts, Phys. Rev. E 64 (2001) 026118.

[9] A.-L. Barabási, R. Albert, Science 286 (1999) 509.

[10] J.-P. Eckmann, E. Moses, Proc. Natl. Acad. Sci. U.S.A. 99 (2002) 5825.

[11] L.A.N. Amaral, A. Scala, M. Barthélémy, H.E. Stanley, Proc. Natl. Acad. Sci. U.S.A. 97 (2000) 149.

[12] M.E.J. Newman, Proc. Natl. Acad. Sci. U.S.A. 98 (2001) 404.

[13] M.E.J. Newman, Phys. Rev. E 64 (2001) 016131.

[14] M. Kuperman, G. Abramson, Phys. Rev. Lett. 86 (2001) 2909.

[15] O. Miramontes, B. Luque, Physica D 168 (2002) 379.

[16] D. Zanette, Phys. Rev. E 65 (2002) 041908.

[17] D.J. Watts, Small Worlds, Princeton University Press, Princeton, 1999.

[18] R.F.I. Cancho, C. Janssen, R.V. Solé, Phys. Rev. E 64 (2001) 046119.

[19] H. Jeong, B. Tombor, R. Albert, Z.N. Oltvai, A.-L. Barabási, R. Albert, Nature (London) 407 (2000) 651.

[20] L.F. Lago-Fernández, R. Huerta, F. Corbacho, J.A. Sigüenza, Phys. Rev. Lett. 84 (2000) 2758.

[21] P.M. Gade, C.K. Hu, Phys. Rev. E 62 (2000) 6409.

[22] J. Jost, M.P. Joy, Phys. Rev. E 65 (2001) 016201.

[23] X. Wang, G. Chen, Int. J. Bifurcat. Chaos 12 (2002) 187.

[24] X. Wang, Int. J. Bifurcat. Chaos 12 (2002) 885.

[25] M. Barahona, L.M. Pecora, Phys. Rev. Lett. 89 (2002) 054101.

[26] V. Belykh, I. Belykh, M. Hasler, Physica D, this issue.

[27] L.M. Pecora, T.L. Carroll, Phys. Rev. Lett. 80 (1998) 2109;

L.M. Pecora, Phys. Rev. E 58 (1998) 347;

K. Fink, G. Johnson, T. Carroll, D. Mar, L. Pecora, Phys. Rev. E 61 (2000) 5080.

[28] D.L. Mills, IEEE Trans. Commun. 39 (1991) 1482.

[29] D.L. Mills, ACM Comput. Commun. Rev. 24 (1994) 16.

[30] D.-G. Holmes, T.A. Lipo, Pulse Width Modulation for Power Converters: Principles and Practice, Wiley-IEEE Press, 2003.

[31] N.N. Bogoliubov, Yu.A. Mitropolsky, Asymptotic Methods in the Theory of Nonlinear Oscillations, Gordon and Breach, New York, 1961.

[32] H. Chernoff, Ann. Math. Statist. 23 (1952) 493-507.

[33] T. Hagerup, C. Rub, Inf. Proc. Lett. 33 (1990) 305. 